\title{
Effect of TTL Parameter Variation on Performance of AODV Route Discovery Process
}

\author{
Puneet Bindra \\ M.tech Scholar \\ SBSSTC, Ferozepur
}

\author{
Jaswinder Kaur \\ A.P (ECE) \\ SBSSTC, Ferozepur
}

\author{
Gurjeevan Singh \\ DIC (ECE) \\ SBSSTC(Polywing), Ferozepur
}

\begin{abstract}
A routing protocol is used to facilitate communication in ad hoc network. The primary goal of such a routing protocol is to provide an efficient and reliable path between a pair of nodes. Routing in Mobile Ad Hoc Networking technology (MANET) is challenging due to its route discovery feature dealing with link failures and to repair the routes in these situations. An ad hoc network is a temporarily infrastructure less network in which nodes can join and leave the network at anytime and are free to move randomly and organize themselves arbitrarily. In MANETs, each node should not only work for itself, but should be cooperative with other nodes. In this paper, we propose a scheme to evaluate effect of Time To Live (TTL) increment and threshold value to analyze route discovery process in Ad hoc on Demand Distance Vector (AODV) routing protocol for proactively less communication overheads. It is simulated using OPNET 14.5 with fixed network area and node density. Our simulation results show that performance of route discovery, link failure and repair mechanism depends on optimal choice of TTL increment and threshold.
\end{abstract}

\section{Keywords}

MANET, OPNET 14.5, Routing Protocol, AODV

\section{INTRODUCTION}

Wireless cellular systems have been in use since 1980s. Future generation wireless systems will require easy and quick deployment of wireless networks. Recent advancements introduced a new type of wireless systems known as mobile ad-hoc networks [1]. During the last couple of years tremendous improvements are made in the research of ad hoc networks. Self configurability and easy deployment feature of the MANET resulted in numerous applications in this modern era. Efficient routing protocols will make MANETs reliable. Now-a-days mobile ad hoc networks have robust and efficient operation in mobile wireless networks as it can include routing functionality into mobile nodes which is more than just mobile hosts and reduces the routing overhead and saves energy for other nodes [2].

AODV enables dynamic, self starting, multi hop, on demand routing for mobile wireless ad hoc networks. A node does not have to discover and maintain a route to another node until the two needs to communicate. When the local connectivity of the mobile node is of interest, each mobile node can become aware of the other nodes in its neighborhood by the use of several techniques, including local broad-casts known as TTL values [3]. Route finding in AODV protocol is based on a route discovery cycle involving a broadcast network search and a unicast reply containing discovered paths. Routing discovery of AODV is mainly divided into two parts, reverse route establishment and forward route establishment [4]
For routing three control messages are used: RREQ (Route Request), RREP (Route Reply), RERR (Route Error). If a node wants to send a packet to a node for which no route is available it broadcasts a RREQ to find one. A RREQ includes a unique identifier, the destination IP address and sequence number, the source IP address and sequence number as well as a hop count initialized with zero and some flags. If a node receives a RREQ which it does not have seen before it sets up a reverse route to the sender. If it does not know a route to the destination it rebroadcasts the updated RREQ especially incrementing the hop count. If it knows a route to the destination it creates a RREP. A RREP contains the destination IP address and sequence number, the source IP address, a time to life, a hop count as well as a prefix only used for subnets and some flags. When a node receives a RREP it checks if the hop counts in the RREP for the emitter of the message is lower than the one in its own routing table or the destination sequence number in the message is higher than the one in its own routing table. It updates its routing table and if it is not the destination it reunicasts the RREP. These nodes are notified with RERR (Route Error) packets when the next-hop link breaks. This packet gets forwarded by each predecessor node to its predecessors, effectively erasing all routes using the broken link [5]. The RERR message can be broadcasted if there are many neighbours who need that information or unicasted if there is only one neighbour. However, iterative unicasting must be considered as a single broadcast RRER message, so that RERR messages per second limit are essential.

AODV does not need any central administrative system to handle the routing process. The paper is structured as follows: a brief overview of route discovery, link failure and repair mechanism is given in Section 2. In Section 3 we present related work in ad hoc networks. Section 4 then enfolds Experimental set up and Results are displayed in section 5 .Section 6 concludes this work and gives an outlook to further possible developments and research topics in this area.

\section{ROUTE DISCOVERY, FAILURE AND LOCAL REPAIR}

When a source node desires to send a message to some destination node, it broadcasts a RREQ packet to its neighbours by expanding ring search method where TTL increment of TTL in RREQ packet is used to forward the request to their neighbours until the destination is located. Each node maintains its own sequence number as well as broadcast ID. Intermediate nodes can reply to the RREQ only if they have a route to the destination whose corresponding destination sequence numbers is greater than or equal to that contained in RREQ. Route request keep propagating until the TTL field reaches zero or the whole connected network is 
covered. Once RREQ reaches the destination, the destination node responds by sending RREP packet back to the neighbours from which it first received the RREQ. As RREP is routed back, nodes along this path set up forward route entries which point to the node from which RREP came. Each route entry is associated with route timer which will delete the entry after its specified life time. Performance of route discovery in AODV largely depends on TTL values of search set which are used sequentially to find desired destinations. If the node can't find a route to destination then the RERR packet is sent to the originator of the packet hop by hop [6]. Link between the nodes can break due to node mobility, high error rate or packet collisions. A mechanism must exist for the nodes to determine link break. Nodes have to trigger the local route repair procedure when they predict that connection is about to break. When a link break in an active route occurs, the node upstream of that break may choose to repair the link locally if the destination was no farther than TTL Threshold hops away. To repair the link break, the node increments the sequence number for the destination and then broadcasts a RREQ for that destination. The node initiating the repair then waits the discovery period to receive RREPs in response to the RREQ. The destination node sends a RREP via selected route with better route and packets are not dropped when route break occurs.

\section{RELATED WORK}

Route discovery is aimed to save resources, reduce route failure and minimize routing overhead.

Existing approaches include [7] ERS (Expanding Ring Search) based enhancement in basic TTL sequence resulting into faster route discovery.

In [6] problem of intermediate route building in AODV has been solved that also enhances the performance of AODV protocol which consists of concept of local recovery with limited TTL value. Evaluation of effects of the route repair is done in [8] which are aimed to efficiently repair the link errors.

To control congestion by applying efficient local route repair method if destination was no farther than maximum repair TTL was proposed in [9].

In this paper, we will focus on a scheme to find optimum values of TTL and evaluate effect of TTL increment and threshold value for faster and reliable route discovery process.

\section{EXPERIMENTAL SET UP}

In the experiment, we have generated a wireless network consisting of 50 nodes randomly placed in $500 \times 500$ square meter area and simulation duration is 300 seconds. Each node is moving with a speed of $10 \mathrm{~m} / \mathrm{s}$. The simulation tool used for analysis is OPNET 14.5 which is highly preferred by researchers [10].It is a synthetic simulation tool to simulate and analyze for network communication and it can provide three tier model, where the top layer is the network layer that reflects the topology of the network, the middle layer is the node layer that is composed of the corresponding protocol models and the bottom layer is the process model that is described by finite state machines.

Depending on total no. of nodes, size of network and diameter of network D, TTL values for expanding ring search are chosen as given in following Table1.
Table 1.TTL Parameters

\begin{tabular}{|l|l|l|l|}
\hline ERS & $\begin{array}{l}\text { TTL } \\
\text { start }\end{array}$ & $\begin{array}{l}\text { TTL } \\
\text { increment }\end{array}$ & $\begin{array}{l}\text { TTL } \\
\text { threshold }\end{array}$ \\
\hline ERS 1 & 1 & 1 & 7 \\
\hline ERS2 & 1 & 1 & 21 \\
\hline ERS3 & 1 & 2 & 7 \\
\hline
\end{tabular}

The simulation parameters used in the scenarios are shown in Table2.

Table 2. Simulation Parameters

\begin{tabular}{|l|l|}
\hline Parameters & Value \\
\hline Simulation Area & $500 \mathrm{~m} \mathrm{X} \mathrm{500m}$ \\
\hline Simulation Time & $300 \mathrm{sec}$ \\
\hline Mobile Node Placement & Random Way Point \\
\hline Buffer Size (BS) & 128000 \\
\hline Packet Size & 1024 Bytes \\
\hline Routing Protocol & AODV \\
\hline No. of Mobile Nodes(MN) & 50 \\
\hline Transmit Power & $0.001 \mathrm{~W}$ \\
\hline Node Traversal Time & $0.04 \mathrm{msec}$ \\
\hline Node Speed & $10 \mathrm{~m} / \mathrm{s}$ \\
\hline Diameter of network (D) & 25 \\
\hline
\end{tabular}

The following metrics are used in this paper for the analysis of AODV Route Discovery Process

Throughput: It is the average rate of successful message delivery over a communication channel. Delay: It represents end-to-end delay which refers to the time taken for a packet to be transmitted across a network from source to destination. Number of Hops per Route: It represents no. of hops in each route to every destination in the route of all nodes in the network.

Total Route Requests Sent: It represents total number of route request packets sent by all nodes in the network during route discovery.

Total Route Errors Sent: It represents total number of route errors sent by all nodes in the network.

\section{RESULTS AND DISCUSSIONS}

In this section, simulation results have been discussed. The performance of route discovery process is analysed in terms of Throughput, Delay, Total Route Requests Sent, Total Route Error Sent and Number of Hops per Route by evaluating effect of TTL increment and TTL threshold. 


\subsection{Throughput}

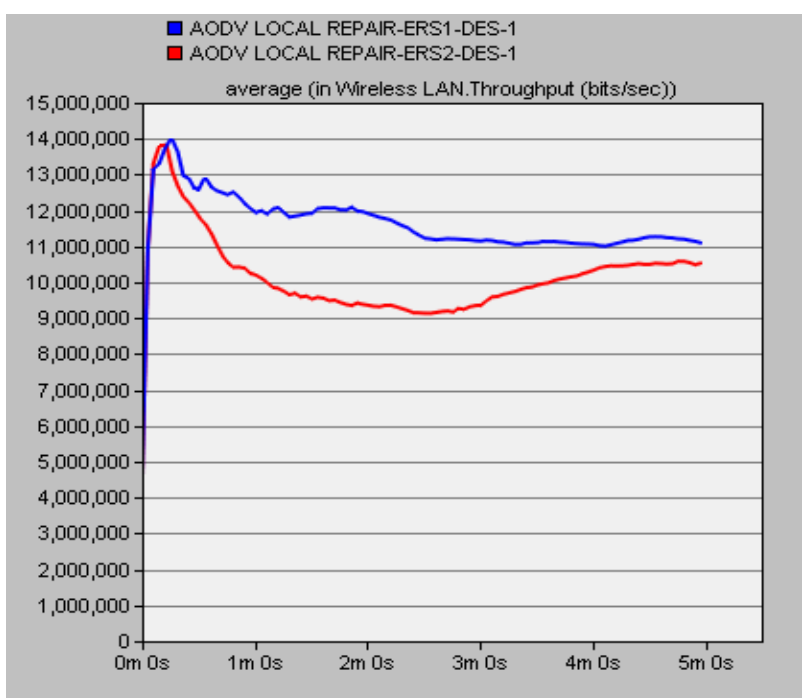

Fig 1: Throughput - varying TTL threshold

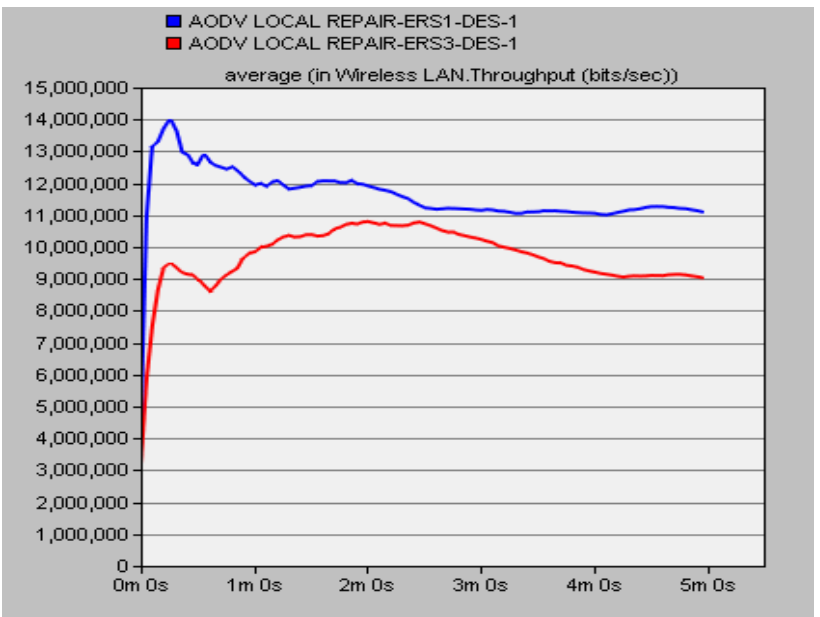

Fig 2: Throughput- varying TTL increment

From figure 1 it is observed that more throughput is obtained in ERS1 than ERS2. Throughput increases with low threshold value as there is less probability of storing stale routes. Large TTL threshold value affects the size of successive rings and decreases throughput. In figure 2 throughput for ERS1 is greater than ERS3. As there is larger number of hops in ERS1, it will result in better throughput in case of less TTL increment value than ERS3. ERS3 has lesser number of hops as TTL increment in ERS3 is 2. ERS3 broadcasts the request again with TTL increment 2 whereas ERS1 broadcasts the route request again with TTL increment 1 so ERS1 has larger number of hops.

\subsection{Delay}

By studying the results in figure 3 large overall delay is observed in ERS2 with greater threshold value. AODV ERS2 suffers more delay because stale routes in route cache consume time for route caching. AODV ERS1 attains lowest routing latency as number of route requests propagating are less in ERS1 as compared to ERS2 because as the route requests propagates more delay increases. In figure 4, large delay is observed in ERS1 which has less increment value than ERS3. Even though ERS1 attains greater throughput but it causes delay due to the neighbor node involvement for making decision.

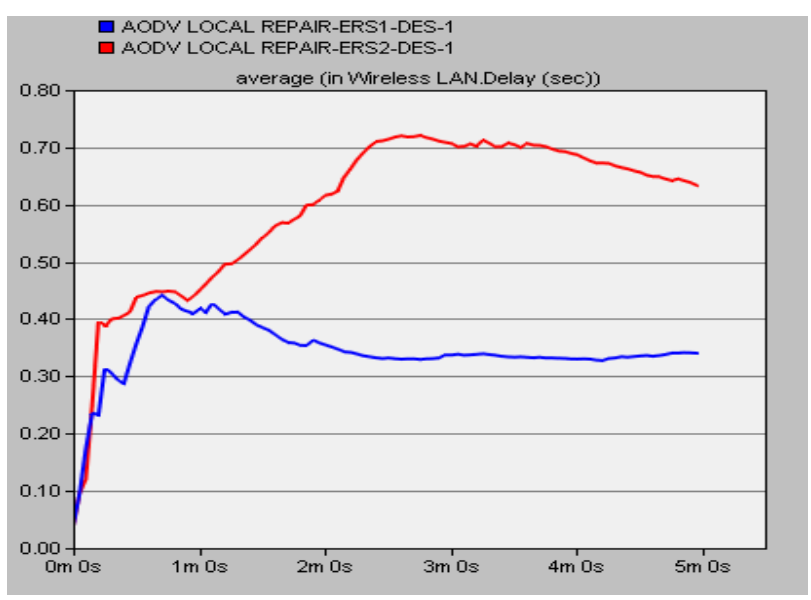

Fig 3: Delay- varying TTL threshold

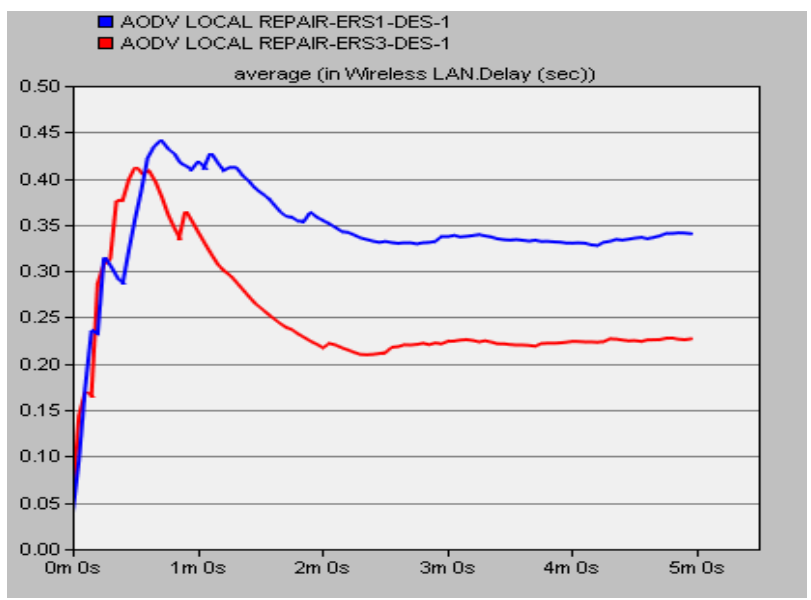

Fig 4: Delay- varying TTL increment

\subsection{Total Route Requests Sent}

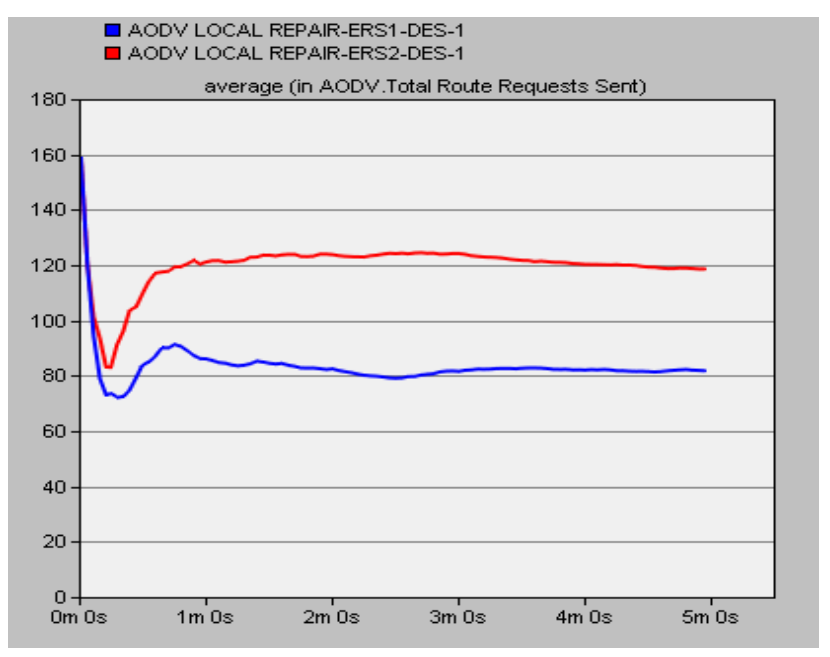

Fig 5: Route Request- varying TTL threshold 
In figure 5, it is observed that less route requests are sent in AODV ERS1 with low threshold value as compared to ERS2. Reducing route requests messages would result in faster and reliable route discovery process. Proportion of RREQ messages increases in ERS2 because of more delay experienced in ERS2.

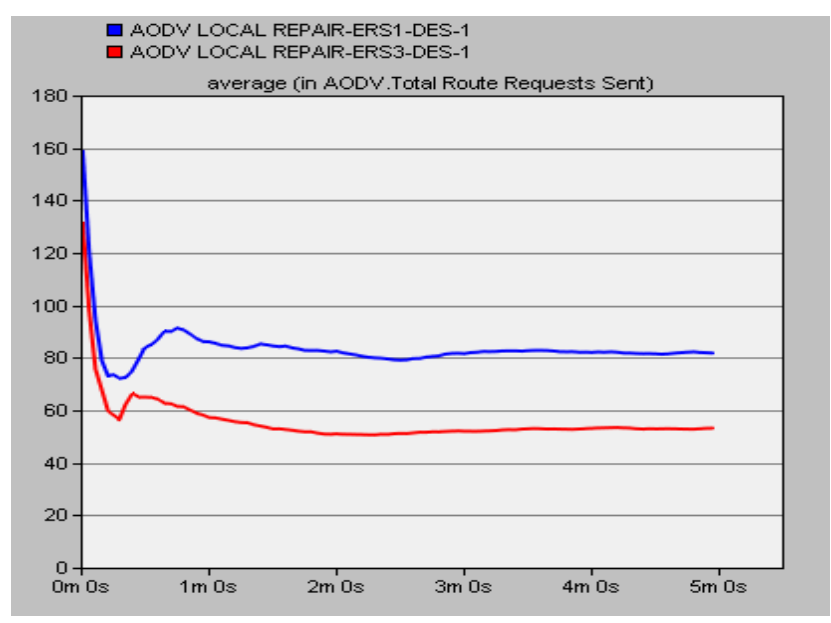

Fig 6: Route Request- varying TTL increment

In figure 6 , it can be seen that more routing requests are generated in ERS1 than ERS3 as the distance between source and destination is greater in ERS1 due to low increment value. When nodes broadcast RREQ messages those messages are received by more nodes and each node receives a greater number of RREQ messages.

\subsection{Number of Hops per Route}

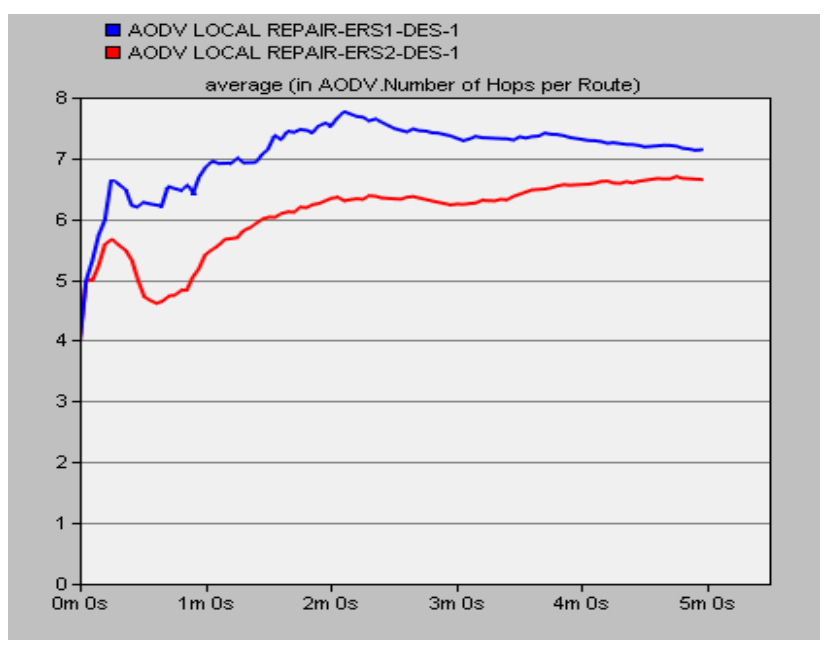

Fig 7: Hops per Route- varying TTL threshold

Number of hops per route is shown in figure 7. Average number of hops experienced is less in AODV ERS2 as less number of hops may represent nodes are close enough to each other in high TTL threshold. Distance between source and destination may be less in high threshold. In figure 8 less number of hops is experienced in ERS3 with greater TTL increment as the distance between source and destination would be less with more increment value.

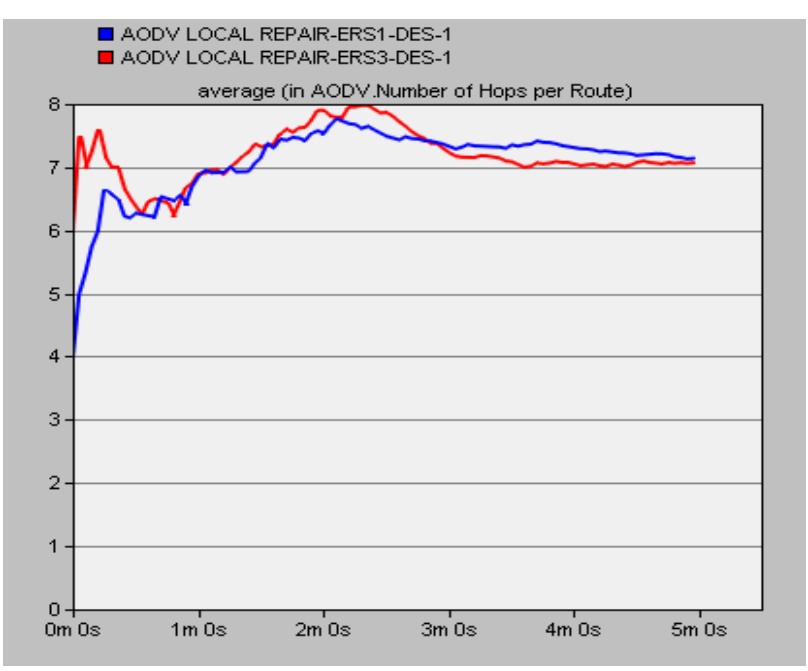

Fig 8: Hops per Route- varying TTL increment

\subsection{Total Route Errors Sent}

From figure 9 it is clear that more number of route errors are sent in AODV ERS 2 and less route errors are experienced in AODV ERS1 with less threshold value. More number of route errors is experienced in AODV ERS2 with greater threshold value. This is because with mobility some destinations can become unreachable or utilize long time to find routes causing route errors to be sent and routes to be removed. To create new routes again new route request need to be originated resulting in delay. In figure 10 more route errors are experienced in ERS1 as when number of hops is more in ERS1 there is high chance of link breakage and local repair attempt will be more than ERS3. So more route errors are generated in ERS1 and so are the routing requests also.

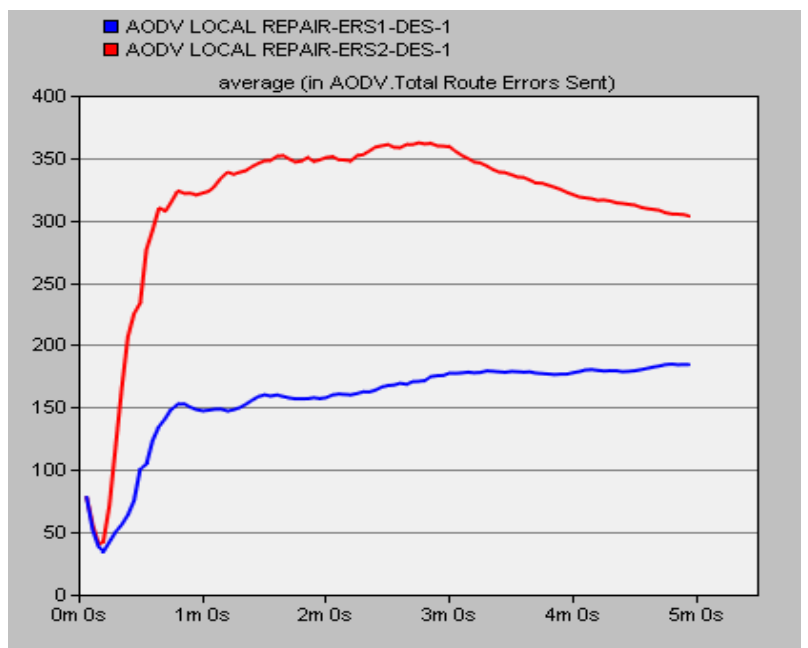

Fig 9: Route Errors- varying TTL threshold 


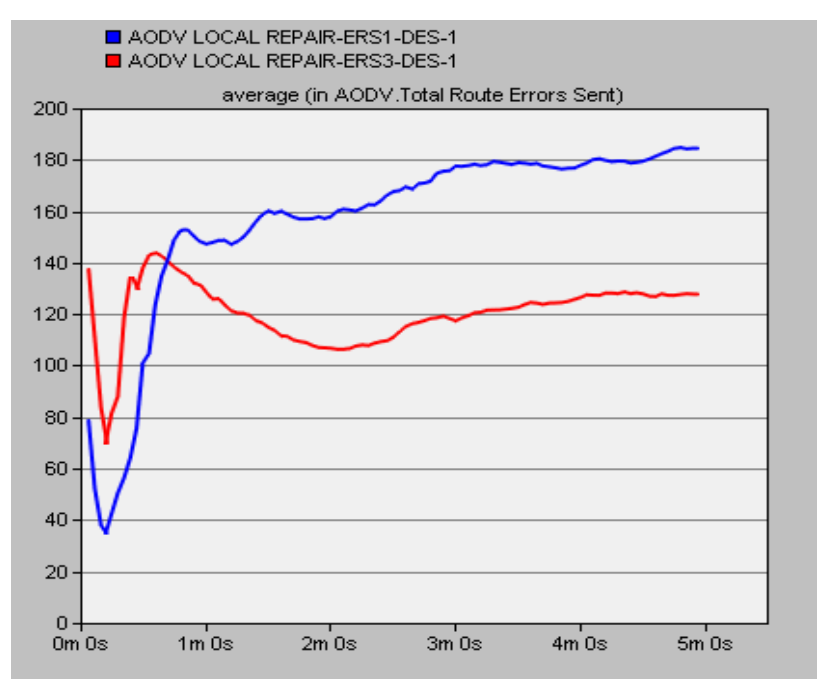

Fig 10: Route Errors - varying TTL increment

\section{CONCLUSION}

In this paper, a scheme is proposed for better route discovery process and route maintenance through local repair. Depending upon diameter and size of network different values of TTL parameters of AODV were chosen. To analyze effects of TTL parameters on route discovery process, effect of TTL increment and TTL threshold were simulated. Simulation results show optimum values of TTL parameters and their effects for different expenses in terms of throughput, delay, no. of route requests sent, No. of route errors sent and no. of hops per route.

\section{REFERENCES}

[1] Pravin Ghosekar, Girish Katkar and Dr. Pradip Ghorpade," Mobile Ad Hoc Networking: Imperatives and Challenges", IJCA, pp 153-158, 2010
[2] Nadia Qasim, Fatin Said and Hamid Aghvami,"Mobile Ad Hoc Networking Protocols Evaluation through Simulation for Quality of Service", IAENG International Journal of Computer Science, 36:1, IJCS_36_1_10

[3] Jyoti Jain, Roopam Gupta and T.K. Bandhopadhaya,"On Demand Local Link Repair Algorithm for AODV Protocol", IJCA, pp 20-25, Vol 35- No.5, December 2011

[4] Lei Guo, Yuhuai Peng, Xingwei Wang, Dingde Jiang and Yinpeng $\mathrm{Yu}$, "Performance evaluation for ondemand routing protocols based on OPNET modules in Wireless Mesh Networks", Computers and Electrical Engineering ,pp 106-114,2011

[5] S. R. Biradar, Koushik Majumder, Subir Kumar Sarkar and Puttamadappa,"Performance Evaluation and Comparison of AODV and AOMDV",IJCSE International Journal on Computer Science and Engineering, Vol. 02, No. 02,pp 373-377, 2010

[6] Anurag Porwal, B.L.Pal,Rohit Maheshwari and Gaurav Kakhani,"Study and Design of New Reactive Routing Protocol Advance AODV for Mobile Ad hoc Networks",IJERA, pp 3195-3204, Vol.2, issue 3, MayJun 2012

[7] Purvi N. Ramanuj and Hiteishi M. Diwanji, "Enhanced Expanding Ring Search Algorithm for AODV”, IJCA, pp 60-64, Jan 2012

[8] Michael Pan, Sheng- Yan Chuang and Sheng -De Wang, "Local Repair Mechanisms for On - Demand Routing in Mobile Ad hoc Networks"

[9] Kishan Singh Rao and Laxmi Shrivastav," Efficient Local Route Repair Method in AODV to Reduce Congestion in Manet",Corona Journal of Science and Technology, pp 35-38, Vol. 1 No. 1, Octuber 2012

[10] Viranch Rajawat,"ELE4NET Communication Networks" ,Department of Electronic Engineering La Trobe University. 